\title{
Formação profissional e conhecimento sobre biossegurança de Auxiliares de Saúde Bucal dos setores público e privado
}

\author{
Geisiane Rodrigues Paes*; Jéssica Teixeira Ramos*; Maiara Medeiros Ronsani**; Thiago Martins \\ Meira** \\ * Estudante de Graduação, Bacharelado em Enfermagem, \\ Universidade do Estado da Bahia \\ ** Mestre em Odontologia, Professor Auxiliar, Bacharelado em \\ Enfermagem, Universidade do Estado da Bahia
}

Recebido em 02/07/2017. Aprovado em 10/09/2017.

\begin{abstract}
RESUMO
$\mathrm{Na}$ Odontologia, o conhecimento de medidas de biossegurança relacionadas ao controle de infecção constituem preocupação constante, já que conhecimentos adquiridos podem ser aplicados para a prevenção, minimização ou eliminação de riscos à saúde. O objetivo deste estudo foi verificar a relação entre a formação profissional e o conhecimento sobre biossegurança de Auxiliares de Saúde Bucal (ASBs) dos serviços público e privado em uma cidade do sudoeste da Bahia. Trata-se de um estudo transversal envolvendo 44 profissionais, sendo 16 do setor público e 28 do setor privado. Utilizou-se questionário semiestruturado com 22 questões. Os dados foram analisados por meio de estatística descritiva e pelo teste Qui-quadrado. A análise dos dados revelou que 45,5\% das ASBs ainda não haviam realizado curso técnico profissionalizante, capacitação ou atualização e 77,3\% não possuíam registro no Conselho Regional de Odontologia. As participantes que haviam recebido algum tipo de informação técnico-científica mostraram saber mais sobre o significado do termo biossegurança $(\mathrm{p}<0,05)$ e realizar com maior frequência a lavagem das mãos entre um paciente $\mathrm{e}$ outro $(p<0,05)$. As profissionais do serviço público mostraram maior conhecimento sobre o significado do termo biossegurança $(\mathrm{p}<0,05)$ e a possiblidade de transmissão de doenças para os pacientes $(p<0,05)$, em comparação às do serviço privado. Observou-se neste estudo que uma melhor formação profissional das ASBs pode interferir positivamente sobre os conhecimentos de biossegurança referentes ao controle de infecção.
\end{abstract}

Descritores: Formação Profissional. Auxiliar de Consultório Dentário. Biossegurança.

\section{INTRODUÇÃO}

No ambiente odontológico, medidas de biossegurança são extremamente importantes, já que se trata de um meio potencialmente infecto em decorrência da presença de fluidos biológicos como saliva, sangue e coleções purulentas. As ações adotadas permitirão o controle de infecções, a proteção da equipe de assistência e dos usuários em saúde e a promoção da consciência sanitária ${ }^{1,2}$. 
Ao longo dos anos, as profissões auxiliares assumiram papel de destaque na divisão do trabalho odontológico ao ajudar os cirurgiõesdentistas (CD) em suas atividades. Tão importante é a participação desses profissionais no espaço odontológico, que em 24 de dezembro de 2008 foi sancionada a lei n. ${ }^{\circ} 11.889$, aprovada pelo Congresso Nacional, que regulamenta o exercício das profissões de Técnico em Saúde Bucal (TSB) e Auxiliar de Saúde Bucal (ASB) no país ${ }^{3}$.

Tendo em vista as condições de trabalho e o crescente número de ASBs, são necessárias medidas que garantam capacitação profissional de qualidade, visando erradicar práticas inadequadas e a reforçar a implementação de medidas de proteção que resultem na redução da ocorrência de acidentes com exposição a materiais biológicos ${ }^{4}$.

Apesar da grande importância do trabalho dos ASBs, nem sempre estes profissionais possuem formação técnica adequada para exercer esta função ${ }^{5}$. Em adição, são ainda muito escassos na literatura estudos científicos brasileiros que verifiquem a relação existente entre a formação profissional e o conhecimento sobre biossegurança desses profissionais, no que diz respeito ao controle de infecção.

Em um estudo realizado na Alemanha ${ }^{6}$, onde se avaliou o cumprimento das recomendações de controle de infecção por CDs e ASBs e o risco de contaminação cruzada no ambiente de trabalho, foi demonstrado que apenas uma pequena percentagem dos profissionais de saúde bucal realizaram a higienização das mãos com as práticas adequadas e que os ASBs cumpriam consideravelmente menos as práticas de controle de infecção.

Devido à importância da atuação dos ASBs nos serviços de saúde público e privado, é necessário que se desenvolvam estudos que permitam a reflexão sobre a formação profissional e a atuação destes profissionais na área da saúde, sobretudo sobre ações que envolvam seus conhecimentos de biossegurança com ênfase nas medidas de controle de infecções, para proteção individual e coletiva.

Nessa perspectiva, o objetivo do estudo foi verificar a relação existente entre formação profissional e conhecimento sobre biossegurança de auxiliares de saúde bucal dos serviços público e privado de um município no sudoeste da Bahia.

\section{METODOLOGIA}

Trata-se de um estudo transversal, realizado com todas as auxiliares de saúde bucal maiores de 18 anos que trabalhavam no setor público e privado em uma cidade do sudoeste da Bahia, que foi aprovado pelo Comitê de Ética em Pesquisa da Universidade do Estado da Bahia (CEPUNEB), sob o parecer 472.837.

Estavam em atividade durante o período do estudo (janeiro e fevereiro de 2015) 18 auxiliares nas Unidades Básicas de Saúde, conforme listagem fornecida pela Secretaria Municipal de Saúde. Não haviam registros prévios a respeito destes profissionais em atividade da rede privada. Sendo assim, o recrutamento dos participantes do referido setor foi realizado por meio de visita aos consultórios devidamente registrados na vigilância sanitária do município, conforme listagem cedida pelo órgão. Assim, verificou-se a existência de 41 profissionais.

O questionário utilizado para a coleta de dados foi um instrumento utilizado por Cortelli $(2012)^{7}$ com algumas adaptações. Trata-se de um questionário semiestruturado com 22 questões sobre formação profissional e conhecimentos básicos de biossegurança. As variáveis analisadas foram o grau de escolaridade, o tempo de trabalho, o nível de formação técnica, assim como o conhecimento sobre biossegurança, riscos de infecção cruzada, doenças infectocontagiosas, vias de transmissão e 
imunização.

Foi solicitada a realização da leitura e a assinatura do Termo de Consentimento Livre e Esclarecido (TCLE), conforme a resolução $\mathrm{n}^{\circ}$ 466/12 do Conselho Nacional de Saúde, além de serem realizados esclarecimentos sobre o mesmo, assim como sobre o sigilo das informações e a preservação da identidade. Os questionários foram entregues aos participantes para o autopreenchimento em local privativo e devolvidos em seguida. Os questionários respondidos foram depositados em um classificador selado, com pequena abertura lateral (simulando uma urna) sendo aberto somente após o término da coleta de todos os dados. Este procedimento impediu o acesso dos pesquisadores aos questionários após cada preenchimento e isso assegurou a preservação da identidade das participantes.

Os dados obtidos foram tabulados no software Microsoft Excel 2010 e analisados por meio do pacote estatístico IBM SPSS 22.0 Statistical Package for the Social Sciences (SPSS Inc., Chicago, USA) com a realização do teste de associação qui-quadrado de Pearson $\left(\mathrm{X}^{2}\right)$ a um nível de significância de $5 \%$.

\section{RESULTADOS}

Da listagem total de consultórios odontológicos (57 estabelecimentos), oito endereços não foram encontrados, nove funcionavam sem auxiliar e em cinco deles não era mais exercida atividade odontológica. Durante a coleta de dados foram encontrados mais seis consultórios privados que foram acrescentados ao estudo, totalizando quarenta e um estabelecimentos.

A taxa de resposta foi de $84,21 \%$ para o setor público $(n=16)$ e $68,29 \%$ o privado $(n=28)$. Três questionários da rede privada foram excluídos por preenchimento incompleto. No setor público uma auxiliar estava em período de férias e uma unidade não foi visitada por localizar-se na zona rural.

As características sociodemográficas dos 44 profissionais de saúde bucal, todas do sexo feminino, são apresentadas na tabela 1 .

As doenças assinaladas pelas participantes do estudo como passíveis de serem adquiridas ou transmitidas durante o exercício de sua profissão estão descritas no gráfico 1.

Quanto à vacinação contra a Hepatite $\mathrm{B}$, observa-se que a maioria $(81,8 \%)$ já havia tomado as três doses recomendadas, $6,8 \%$ não e $9,1 \%$ não sabiam ou não lembravam. $61,4 \%$ das entrevistadas estavam vacinadas contra a gripe e $34,1 \%$ não haviam vacinado. Não foi observada associação entre o setor de trabalho (público ou privado) e vacinação.

Sobre a formação profissional, 37,5\% $(\mathrm{n}=6)$ das profissionais do setor público e $53,8 \%$ $(\mathrm{n}=14)$ das profissionais do setor privado não haviam realizado nenhum curso técnico, profissionalizante, capacitação ou atualização para desenvolver esta atividade profissional. A respeito da inscrição regular no Conselho Regional de Odontologia, 62,5\% ( $\mathrm{n}=10)$ da rede pública e $85,7 \%(n=24)$ da rede privada não possuíam registro. Das profissionais estudadas, $87,5 \%(n=14)$ da rede pública e $35,7 \%(n=10)$ já haviam feito curso ou palestra sobre biossegurança na área da saúde.

As características quanto ao conhecimento sobre biossegurança das auxiliares de saúde bucal estão apresentadas na tabela 2. As ASBs do serviço público mostraram melhor conhecer o significado do termo biossegurança em relação às do serviço privado, bem como realizaram com maior frequência curso, palestra e treinamento sobre biossegurança $(\mathrm{p}<0,05)$. A possibilidade de transmissão de doenças para os pacientes durante a atividade profissional era melhor conhecida pelas profissionais do setor público $(\mathrm{p}<0,05)$. 
Tabela 1. Características sociodemográficas das auxiliares de saúde bucal

\begin{tabular}{lcc}
\hline Variável & $\mathbf{n}$ & $\%$ \\
\hline Setor & & \\
Público & 16 & 36,4 \\
Privado & 28 & 63,6 \\
Idade & & \\
18 a 25 anos & 12 & 27,3 \\
26 a 35 anos & 14 & 31,8 \\
36 a 45 anos & 13 & 29,5 \\
46 a 55 anos & 3 & 11,4 \\
\hline & & \\
Sexo & - & - \\
Masculino & 44 & 100 \\
Feminino & & \\
& & \\
Escolaridade & 1 & 2,3 \\
Ensino Fundamental Incompleto & 2 & 4,5 \\
Ensino Fundamental Completo & 1 & 2,3 \\
Ensino Médio Incompleto & 30 & 68,2 \\
Ensino Médio Completo & 6 & 13,6 \\
Ensino Superior Incompleto & 3 & 6,8 \\
Ensino Superior Completo & &
\end{tabular}

\section{Tempo de trabalho em consultório Odontológico}

Menos de 1 ano

De 1 a 2 anos

De 3 a 5 anos

De 5 a 10 anos

De 10 a 20 anos

Mais de 20 anos
6

8

7

14

8

1
13,6

18,2

15,9

31,8

18,2

2,3

\section{Possui formação profissional com registro no Conselho}

\section{Regional de Odontologia (CRO)}

Não

Sim, auxiliar em saúde bucal

Sim, técnico em saúde

bucal/técnico em higiene bucal

Sim, técnico em prótese dentária

Sim, auxiliar em prótese dentária 


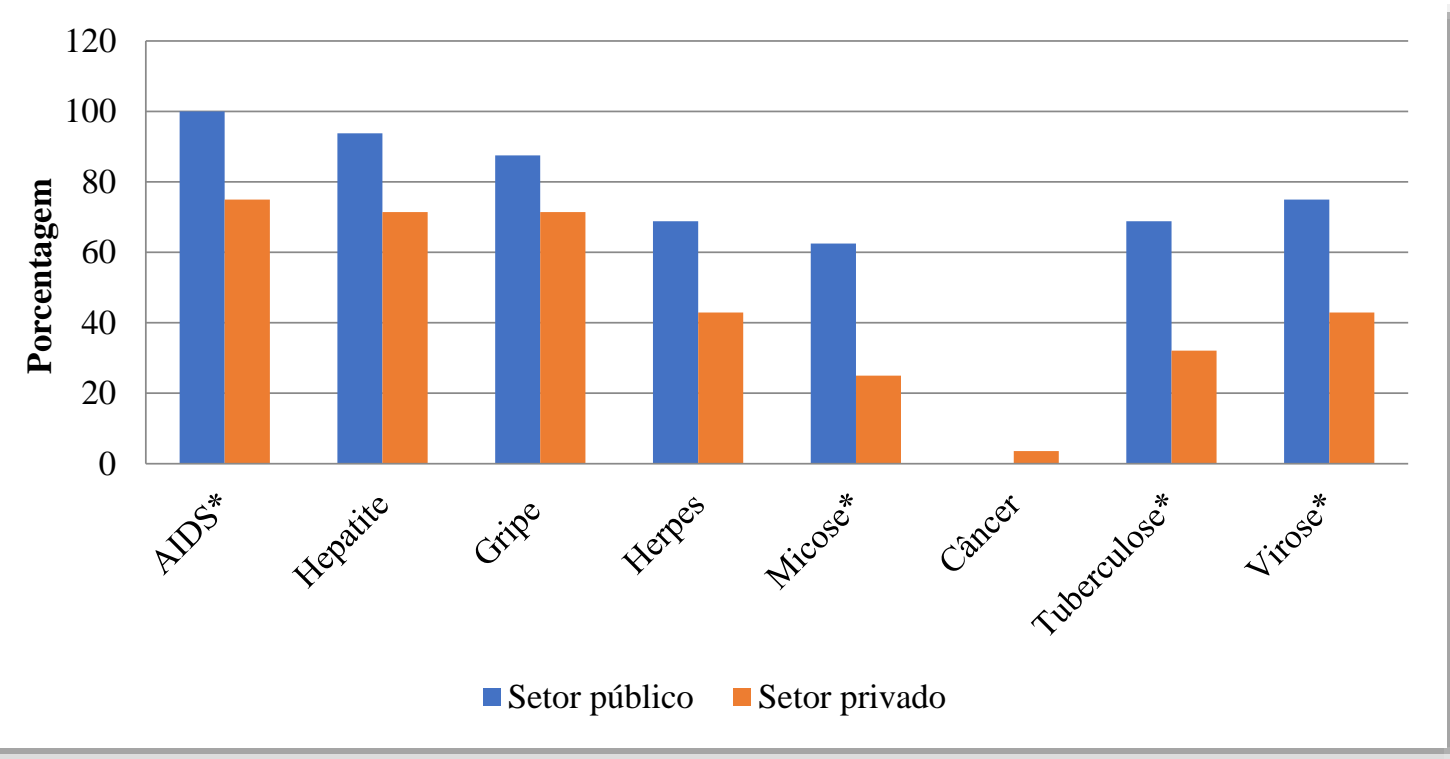

Gráfico 1. Distribuição das ASBs dos setores público e privado quanto ao conhecimento sobre doenças que podem ser adquiridas ou transmitidas no ambiente odontológico, ${ }^{*} \mathrm{p}<0,05$

Apesar de todas as entrevistadas usarem algum tipo de equipamento de proteção individual durante o trabalho, o serviço público utiliza com mais frequência os EPIs, com destaque para o uso de gorro $(\mathrm{p}<0,05)$ (gráfico 2).

As profissionais que já realizaram algum curso técnico, profissionalizante, capacitação ou atualização para desenvolver as atividades de auxiliar de consultório dentário ( $\mathrm{n}=24)$ também realizaram algum curso sobre biossegurança na área da saúde $(\mathrm{p}<0,05)$. Essas profissionais que já receberam algum tipo de informação técnicocientífica $(n=24)$ também sabem mais sobre o significado do termo biossegurança $(\mathrm{p}<0,05)$ e realizam com maior frequência a lavagem das mãos entre um paciente e outro $(\mathrm{p}<0,05)$.

As ASBs que dizem se sentir seguras para exercer sua profissão com o conhecimento que possuem sobre biossegurança são as profissionais que já realizaram algum curso, palestra ou treinamento sobre biossegurança na área da saúde $(\mathrm{p}<0,05)$ e as que conhecem o termo biossegurança $(\mathrm{p}<0,05)$.

\section{DISCUSSÃO}

A qualificação técnica tem um importante resultado para maior eficiência, compreensão e qualidade no desenvolvimento das atividades na clínica e no consultório, como atividades de biossegurança ${ }^{8}$. Nesta pesquisa, as profissionais que realizaram algum curso técnico, profissionalizante, capacitação ou atualização para desenvolver as atividades de auxiliar de consultório dentário mostraram maior conscientização sobre o significado do termo biossegurança $(p<0,05)$ e realizam com maior frequência a lavagem das mãos entre um paciente e outro $(\mathrm{p}<0,05)$. Com base nesses dados, inferese que uma melhor formação profissional pode refletir de forma positiva no conhecimento sobre biossegurança de ASBs e permitir a prática mais segura de sua profissão, sobretudo priorizando medidas universais básicas como a lavagem das mãos entre os procedimentos. 
Tabela 2. Conhecimentos sobre biossegurança das ASBs dos serviços público e privado

\begin{tabular}{|c|c|c|c|}
\hline Variáveis & $\begin{array}{c}\text { Setor Público } \\
\mathrm{n}(\%)\end{array}$ & $\begin{array}{c}\text { Setor Privado } \\
n(\%)\end{array}$ & Valor $\mathbf{p}$ \\
\hline \multicolumn{4}{|c|}{ Sabe o que significa o termo biossegurança? } \\
\hline Não & $2(12,5)$ & $13(46,4)$ & \\
\hline Sim & $14(87,5)$ & $15(53,6)$ & $0,022 *$ \\
\hline \multicolumn{4}{|c|}{ Acredita que pode adquirir doença de pacientes? } \\
\hline Não & 0 & $5(17,9)$ & \\
\hline Sim & $16(100)$ & $21(75)$ & \\
\hline Não sei / Não lembro & 0 & $1(4)$ & 0,191 \\
\hline \multicolumn{4}{|c|}{ Acredita que pode transmitir doença para os pacientes? } \\
\hline Não & $2(12,5)$ & $19(67,9)$ & \\
\hline Sim & $14(87,5)$ & $7(25)$ & \\
\hline Não sei / Não lembro & 0 & $2(7,1)$ & $0,000 *$ \\
\hline \multicolumn{4}{|c|}{ Sabe para que serve a esterilização dos materiais? } \\
\hline Não & 0 & $1(3,6)$ & \\
\hline Sim & $16(100)$ & $26(92,9)$ & 0,550 \\
\hline Omisso & & $1(3,6)$ & \\
\hline \multicolumn{4}{|c|}{$\begin{array}{l}\text { Sabe qual é a forma correta de se descartar os materiais } \\
\text { perfurocortantes? }\end{array}$} \\
\hline Não & 0 & $3(12)$ & \\
\hline Sim & $16(100)$ & $23(88)$ & 0,358 \\
\hline Não sei/ Não lembro & & $1(3,6)$ & \\
\hline Omisso & & $1(3,6)$ & \\
\hline \multicolumn{4}{|c|}{$\begin{array}{l}\text { Sente-se seguro (a) para exercer sua profissão com seu } \\
\text { conhecimento sobre biossegurança? }\end{array}$} \\
\hline Não & 0 & $5(17,9)$ & \\
\hline Sim, em partes & $8(50)$ & $13(46,4)$ & \\
\hline Sim, completamente & $8(50)$ & $8(28,6)$ & \\
\hline Não sei/ Não lembro & 0 & $1(3,6)$ & 0,257 \\
\hline Omissos & & $1(3,6)$ & \\
\hline \multicolumn{4}{|c|}{ Já fez algum curso sobre biossegurança na área da saúde? } \\
\hline Não & $2(12,5)$ & $18(64,3)$ & \\
\hline Sim & $14(87,5)$ & $10(35,7)$ & \\
\hline Não sei/ Não lembro & 0 & 0 & $0,001 *$ \\
\hline \multicolumn{4}{|c|}{ Lava as mãos entre um paciente e outro? } \\
\hline Não & 0 & 0 & \\
\hline Sim, às vezes & $2(12,5)$ & $5(17,9)$ & \\
\hline Sim, entre todos os paciente & $14(87,5)$ & $22(78,6)$ & 0,652 \\
\hline Omissos & & $1(3,6)$ & \\
\hline \multicolumn{4}{|c|}{ Formas de adquirir ou transmitir doença? } \\
\hline Saliva & $12(80)$ & $18(64,3)$ & 0,285 \\
\hline Sangue & $14(93,3)$ & $27(96,4)$ & 0,646 \\
\hline $\mathrm{Ar}$ & $11(73,3)$ & $14(50)$ & 0,139 \\
\hline
\end{tabular}

*resultado estatisticamente significante 


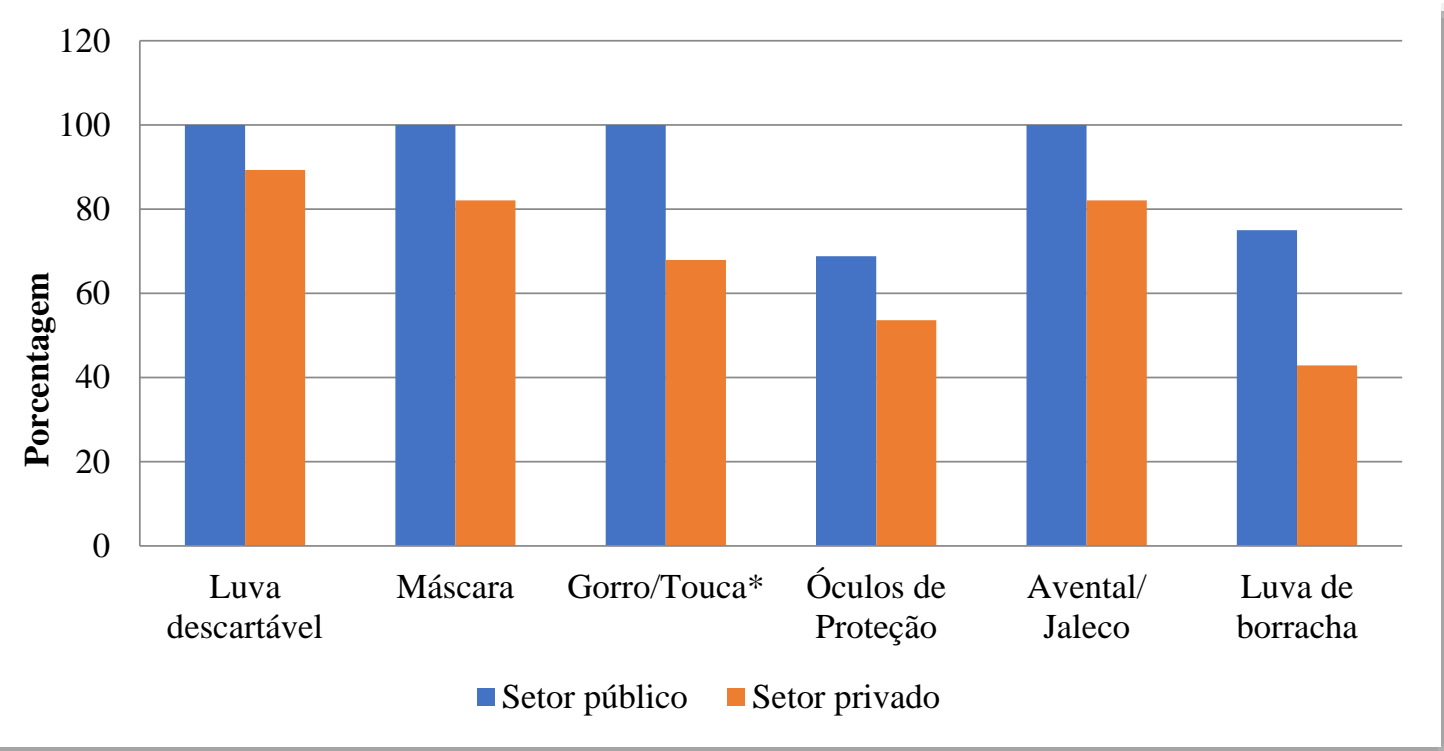

Gráfico 2. Distribuição das ASBs dos setores público e privado quanto ao uso de equipamentos de proteção individual (EPIs), ${ }^{*} \mathrm{p}<0,05$

Por outro lado, uma parcela importante de profissionais deste estudo não possuíam nenhum tipo de formação técnica. Estes resultados nos levam a questionar se estas profissionais sem nenhuma formação técnica específica estão tendo uma percepção adequada sobre os riscos a que estão sendo expostas e a susceptibilidade a estes riscos em seu ambiente de trabalho.

Em outro estudo com $\mathrm{ASBs}^{5}$, apenas $10 \%$ dos auxiliares tinham curso especializado para a função, enquanto que $81 \%$ haviam sido treinados pelo CDs. Durante a formação, os auxiliares adquirem habilidades técnicas específicas, através de cursos oferecidos pelos Centros de Ensino Técnico devidamente reconhecidos pelo Ministério da Educação, por isso o CD incorre em um equívoco ao treinar os auxiliares contribuindo para o aumento do número de profissionais trabalhando sem a devida qualificação ${ }^{9}$. Autores ${ }^{10}$ justificam tal atitude dos cirurgiões dentistas ao dizer que é sabido que não existem muitas escolas técnicas que propiciam a capacitação de profissionais auxiliares e, como o tratamento odontológico exige que todos os profissionais envolvidos estejam tecnicamente preparados, uma das saídas buscadas pelos CDs é o treinamento oferecido dentro do consultório.

Também foi verificado no presente estudo que as ASBs que se sentem seguras para exercer sua profissão com o conhecimento que possuem sobre biossegurança, são as profissionais que conhecem este termo e as que já realizaram algum curso, palestra ou treinamento sobre esta temática na área da saúde $(\mathrm{p}<0,05)$. A literatura ${ }^{11}$ mostra que profissionais de saúde que já fizeram curso de biossegurança também realizaram com maior frequência alguma atualização na sua área profissional, assim como utilizaram EPIs, antissépticos e descontaminação de instrumentos. Novamente verifica-se que uma melhor formação profissional pode permitir que práticas seguras sejam realizadas dentro do ambiente de saúde, como o consultório odontológico. 
A cidade onde desenvolveu-se o presente estudo situa-se distante das maiores áreas urbanas do estado e não há com frequência cursos de capacitação para ASBs. Apesar disso, os dados relatados são semelhantes aos encontrados em estudos realizados em outras cidades do Brasil que não possuem esta limitação, apresentando taxas de $71 \%$ e $61,7 \%$ de profissionais sem a devida inscrição ${ }^{5,10}$. Isso mostra uma realidade nacional que merece atenção por parte de todos os profissionais da Odontologia.

A formação profissional deficiente neste setor pode acarretar em condutas errôneas importantes, especialmente no que diz respeito a práticas de biossegurança mal executadas, a partir de um conhecimento não adquirido. Em adição, constitui infração ética utilizar-se de serviços prestados por profisssionais não habilitados legalmente ou por profissionais da área odontológica não regularmente inscritos no CRO de sua jurisdição, conforme estabelece o inciso IX do artigo $13^{\circ}$ do Código de Ética Odontológica $(\mathrm{CFO})^{12}$.

Pesquisadores $^{13}$ identificaram que as auxiliares de saúde bucal da rede pública usavam com maior frequência os EPIs em comparação à rede privada e sugerem que cursos de treinamento e o fornecimento dos equipamentos aos profissionais do setor público podem justificar essa situação. Neste estudo, o conhecimento superior das ASBs do serviço público, relacionado a medidas de biossegurança no controle de infecções, pode refletir do fato de que um maior número de profissionais desta rede já havia realizado cursos, palestras e treinamento sobre biossegurança $(\mathrm{p}<0,05)$. No estudo de Uchida et al. (2016) ${ }^{14}$, foi identificado no grupo de TSBs o entendimento da importância de realizar cursos de capacitação e esses autores salientam a necessidade de as prefeituras promoverem cursos de capacitação para estes profissionais de forma permanente, já que existe essa demanda no serviço.

A formação profissional dos auxiliares no cenário odontológico atual deve ser amplamente discutida visando também compreender os desafios de interpretar as verdadeiras competências desses profissionais nos serviços de saúde $^{15}$. O TSB e o ASB representam categorias profissionais diretamente envolvidas com atividades que oferecem riscos biológicos à saúde, bem como com competências para desenvolver ações de prevenção de riscos ambientais e sanitários. Apesar da obrigatoriedade de registro no CRO para o TSB e o ASB prevista por lei ( $\left.{ }^{\circ} 11.889 / 2008\right)$, no presente estudo, a grande maioria das auxiliares não era registrada e uma parte considerável não possuía curso técnico, profissionalizante, capacitação ou atualização para desenvolver esta atividade profissional. Diante disso, percebe-se também a necessidade de maior investigação dos fatores que dificultam o cumprimento das normas vigentes, bem como do fortalecimento da discussão desta temática para maior conscientização, incentivo às formações profissionais, facilitando o cumprimento da legislação de forma integral.

Como limitação deste estudo pode-se referir o desenho proposto, pois não há a possibilidade de estabelecer relação causal entre as variáveis estudadas. Entretanto, tal delineamento permitiu verificar a distribuição e características das variáveis investigadas nesta população.

\section{CONCLUSÃO}

A formação profissional das ASBs deste estudo, no que se refere à realização de curso, capacitação e atualização, está associada de maneira positiva sobre seus conhecimentos de biossegurança especificamente relacionados ao controle de infecção. 
O número considerável de profissionais que não conhecem o termo biossegurança e que nunca realizaram alguma forma de capacitação sobre essa temática na área da saúde, além do baixo número de profissionais com registro no CRO, sugere uma grande necessidade de qualificação técnica para desenvolver com qualidade os serviços em saúde entre as ASBs do município estudado, minimizando assim os riscos à saúde dos indivíduos.

\section{AGRADECIMENTOS}

Agradecemos o apoio do Programa de Iniciação Científica PICIN/UNEB.

\section{ABSTRACT \\ Professional training and knowledge on biosafety by Oral Health Assistants of the public and private sectors}

In dentistry, knowledge of biosafety measures related to infection control is a constant concern, since acquired knowledge can be applied to the prevention, minimization, or elimination of health risks. The objective of this study was to verify the relationship between professional training and biosafety knowledge of Oral Health Assistants (OHAs) of the public and private sectors in a city in southwestern Bahia. This is a cross-sectional study involving 44 professionals, 16 from the public sector and 28 from the private sector. A semi-structured questionnaire with 22 questions was used. Data were analyzed using descriptive statistics and a chi-square test. Data analysis revealed that $45.5 \%$ of the OHAs had not yet completed a vocational technical training or refresher course, and $77.3 \%$ had not registered with the Regional Council of Dentistry. Participants who had received some kind of technical-scientific information showed more knowledge about the meaning of the term biosafety $(p<0.05)$ and performed handwashing more frequently between patients $(p<0.05)$. The public sector professionals showed greater knowledge about the meaning of the term biosafety $(\mathrm{p}<0.05)$ and the possibility of disease transmission to patients $(\mathrm{p}<0.05)$ compared to those in the private sector. It was observed in this study that better professional training of the OHAs can positively influence the knowledge of biosafety regarding the control of infection.

Descriptors: Professional Training. Dental Professionals. Exposure to Biological Agents.

\section{REFERÊNCIAS}

1. Diniz DN, Bento PM, Pereira M, Pereira JV, Silva DF, Costa M, et al. Avaliação do conhecimento sobre biossegurança em radiologia pelos alunos do curso de Odontologia da Universidade Estadual da Paraiba. Arq Ciênc Saúde. 2009;16(4):166-9.

2. Krieger D, Bueno R, Gabardo MCL. Perspectivas de biossegurança em odontologia. Rev Gestão Saúde. 2010;1(2):110.

3. Frazão P, Narvai PC. Lei n. ${ }^{\circ} 11.889 / 2008$ : avanço ou retrocesso nas competências do técnico em saúde bucal? Trab Educ Saúde. 2011;9(1):109-23

4. Duarte Filho ESD, Alves GG, Pinheiro FHdSL, de Lima Martelli PJ. Os riscos ocupacionais dos Auxiliares em Saúde Bucal. CATUSSABA-ISSN 2237-3608. 2012;1(2) :57-64.

5. Paranhos LR, Ricci ID, Tomasso S, Siqueira DF. Análise da relação entre o cirurgiãodentista e o pessoal auxiliar. Rev Odonto Ciência. 2008;23(4):365-70.

6. Mutters NT, Hägele U, Hagenfeld D, Hellwig E, Frank U. Compliance with infection control practices in an university hospital dental clinic. GMS Hyg Infec Control. 2014;9(3). DOI: 10.3205/dgkh000238

7. Cortelli AFD. Procedimentos de biossegurança adotados por profissionais prestadores de serviços de manicure, pedicure, tatuagem, piercing e maquiagem definitiva no município de Jacareí-SP [dissertação] São Paulo: Universidade de São 
Paulo; 2012.

8. Silva MPd, Costa GS, Crispim ZAMdP, Castro EA, Cerqueira MBR, Garibalde E. Views of oral health teams in northern Minas Gerais on the training of NHS oral health technicians. Trab Educ Saúde. 2014;12(1):69-85.

9. Leite DFBM, Farias IAP, Costa AdPCd, Barbosa LEdO, Muniz IdAF, Muniz IdAFM. Perfil socioeconômico dos alunos concluintes dos cursos de auxiliar e técnico em saúde bucal. Rev Odontol UNESP. 2012;41(2):95-101.

10. Silva RFd, Monini AdC, Valladares Neto J, Francesquini Júnior L, Daruge Júnior E. Percepção dos auxiliares odontológicos na clínica ortodôntica quanto aos seus limites de atuação profissional. Rev Dental Press Ortod Ortop Facial. 2009;14(3):34-39.

11. da Silva GS, de Almeida AJ, de Paula VS, Villar LM. Conhecimento e utilização de medidas de precaução-padrão por profissionais de saúde. Esc Anna Nery. 2012;16(1):103-10.

12. BRASIL. Conselho Federal de Odontologia. Código de Ética Odontológica, 2012. Resolução CFO-118/2012.20. (Acesso em 05 jan. 2017). Disponível em: http://www. cropr.org.br/uploads/arquivo/6e78019d4c01 c2576de61febb33ff295.pdf.

13. Rodrigues VC. Hepatite B no Município de Ribeirão Preto (SP): um estudo envolvendo cirurgiões-dentistas e auxiliares odontológicos [dissertação]. Ribeirão Preto: Universidade de São Paulo; 2002.

14. Uchida TH, Terada RSS, Tenuta LMA, Fujimaki M. Práticas preventivas realizadas por Técnicos em Saúde Bucal: um estudo qualitativo. Rev ABENO. 2016;16(1):11024.

15. Zanetti CHG, Mendonça MHMd, Oliveira JAAd. Divisão do trabalho odontológico em perspectiva: desafio de interpretar as competências dos técnicos. Trab Educ Saúde. 2012;10(2):195-222.

Correspondência para:

Thiago Martins Meira

e-mail: tmeira@uneb.br

Universidade do Estado da Bahia

Departamento de Educação (DEDC)

Campus XII - Avenida Vanessa Cardoso \&

Cardoso, $\mathrm{s} / \mathrm{n}^{\mathrm{o}}$, Bairro Ipanema

46.430-000 Guanambi/BA 